\title{
Using electronegativity and hardness to test density functionals
}

\author{
Moltved, Klaus August; Kepp, Kasper Planeta
}

Published in:

Journal of Chemical Physics

Link to article, DOI:

$10.1063 / 5.0006189$

Publication date:

2020

Document Version

Publisher's PDF, also known as Version of record

Link back to DTU Orbit

Citation (APA):

Moltved, K. A., \& Kepp, K. P. (2020). Using electronegativity and hardness to test density functionals. Journal of Chemical Physics, 152(24), [244113]. https://doi.org/10.1063/5.0006189

\section{General rights}

Copyright and moral rights for the publications made accessible in the public portal are retained by the authors and/or other copyright owners and it is a condition of accessing publications that users recognise and abide by the legal requirements associated with these rights.

- Users may download and print one copy of any publication from the public portal for the purpose of private study or research.

- You may not further distribute the material or use it for any profit-making activity or commercial gain

- You may freely distribute the URL identifying the publication in the public portal

If you believe that this document breaches copyright please contact us providing details, and we will remove access to the work immediately and investigate your claim. 


\section{Using electronegativity and hardness to test density functionals}

Cite as: J. Chem. Phys. 152, 244113 (2020); https://doi.org/10.1063/5.0006189

Submitted: 01 March 2020 . Accepted: 05 June 2020. Published Online: 24 June 2020

Klaus A. Moltved (D), and Kasper P. Kepp (i)

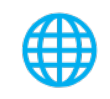




\title{
Using electronegativity and hardness to test density functionals
}

\author{
Cite as: J. Chem. Phys. 152, 244113 (2020); doi: 10.1063/5.0006189 \\ Submitted: 1 March 2020 - Accepted: 5 June 2020 • \\ Published Online: 24 June 2020
}

Klaus A. Moltved (D) and Kasper P. Kepp ${ }^{\text {a) }}$

AFFILIATIONS

Technical University of Denmark, DTU Chemistry, Building 206, 2800 Kgs. Lyngby, Denmark

a) Author to whom correspondence should be addressed: kpj@kemi.dtu.dk. Tel.: +045 45252409

\begin{abstract}
Density functional theory (DFT) is used in thousands of papers each year, yet lack of universality reduces DFT's predictive capacity, and functionals may produce energy-density imbalances. The absolute electronegativity $(\chi)$ and hardness $(\eta)$ directly reflect the energy-density relationship via the chemical potential $\partial \mathrm{E} / \partial \mathrm{N}$ and we thus hypothesized that they probe universality. We studied $\chi$ and $\eta$ for atoms $\mathrm{Z}=1-36$ using 50 diverse functionals covering all major classes. Very few functionals describe both $\chi$ and $\eta$ well. $\eta$ benefits from error cancellation, whereas $\chi$ is marred by error propagation from IP and EA; thus, almost all standard GGA and hybrid functionals display a plateau in the $\mathrm{MAE}$ at $\sim 0.2 \mathrm{eV}-0.3 \mathrm{eV}$ for $\eta$. In contrast, variable performance for $\chi$ indicates problems in describing the chemical potential by DFT. The accuracy and precision of a functional is far from linearly related, yet for a universal functional, we expect linearity. Popular functionals such as B3LYP, PBE, and revPBE perform poorly for both properties. Density sensitivity calculations indicate large density-derived errors as occupation of degenerate $\mathrm{p}$ - and d-orbitals causes "non-universality" and large dependency on exact exchange. Thus, we argue that performance for $\chi$ for the same systems is a hallmark of an important aspect of universality by probing $\partial \mathrm{E} / \partial \mathrm{N}$. With this metric, B98, B97-1, PW6B95D3, MN-15, rev-TPSS, HSE06, and APFD are the most "universal" among the tested functionals. B98 and B97-1 are accurate for very diverse metal-ligand bonds, supporting that a balanced description of $\partial \mathrm{E} / \partial \mathrm{N}$ and $\partial \mathrm{E}^{2} / \partial \mathrm{N}^{2}$, via $\chi$ and $\eta$, is probably a first simple probe of universality.
\end{abstract}

Published under license by AIP Publishing. https://doi.org/10.1063/5.0006189

\section{INTRODUCTION}

Due to its combined computational speed and general accuracy, Density Functional Theory (DFT) is the main methodology used to study electronic structure of larger molecular systems, in which tens of thousands of papers use the methods every year. ${ }^{1,2}$ Often it is the only reasonable option due to the scaling of central processing unit (CPU) requirements with the system size for more accurate methods. ${ }^{2-5}$ However, the acronym "DFT" covers an enormous range of functionals of distinct philosophies and designs, with different physical conditions fulfilled, parameterization ranges, and mathematical forms. ${ }^{2,3,6}$ This fragmentation prevents comparison between studies and obscures the significance of conclusions based on a few functionals, since tests of sensitivity to method choice are rare despite the necessity of performing them. ${ }^{4}$ Separating error contributions beyond the functional itself from, e.g., entropy, zero-point energies, relativistic effects, dispersion effects, or basis set deficiencies is a necessary step in this process. $^{4,7-9}$

After error analysis, we can search for a "universal functional," $10-12$ which per definition minimizes the energy in a constrained search, ${ }^{11}$ but its mathematical structure is elusive. However, it can be defined by its consistency as a pure functional that satisfies all possible fundamental restraints, such as tails of the density, nuclear cusps, virial, ${ }^{13}$ and coordinate scaling, ${ }^{14}$ or by its accuracy as a functional that performs accurately across the broadest ranges of properties of molecular systems by mimicking both the ground state energy and density of all relevant external potentials. ${ }^{12}$ These definitions are not mutually exclusive. Straying ${ }^{15}$ can be defined as imbalances in a thermochemical cycle of trial densities, either along the density or energy path [Fig. 1(a)]. ${ }^{16}$ Some prefer less parameterized, "non-empirical" functionals that satisfy physical bounds such as PBE, TPSS, and SCAN, ${ }^{6,17,18}$ whereas others prefer careful and extensive parameterization toward high-quality 
thermochemical data. ${ }^{19,20}$ Regardless of philosophy, the functional form is central, and different "rungs" represent increasing mathematical complexity. ${ }^{4,21,22}$

An important, but not sufficient, requirement for universality is that the energy and density are described in a balanced way. ${ }^{1}$ One may assume that less empirical functionals are more energydensity balanced [i.e., closer to the central diagonal line of Fig. 1(a)] relatively to empirical functionals that have not considered this balance explicitly, as so far the case. Medvedev et al. ${ }^{15}$ recently used atomic ions to identify energy-density imbalances. Although they did not actually compare the energies and densities for the same systems, ${ }^{25}$ their work and that of Burke ${ }^{26,27}$ show that the balance between density-derived $\left[\Delta E_{\mathrm{D}}{ }^{\prime}\right.$ in Fig. $\left.1(\mathrm{a})\right]$ and functional-derived errors $\left(\Delta E_{\mathrm{F}}{ }^{\prime}\right)$ is important [please note that as written in Fig. 1(a), $\Delta E_{\mathrm{D}}{ }^{\prime}<0$ and $\left.\Delta E_{\mathrm{F}}{ }^{\prime}>0\right]$.

There are many ways to estimate density errors: at the nucleus, at some radial distance, at some point in space, at the tail, for a sum of points, or the density-weighted root-mean-squared deviation from the "exact" density over a grid; this makes error estimates $^{15}$ very conditional. ${ }^{25}$ Instead, Burke suggested to measure density errors via their impact on the energy relative to the exact density, giving a single, unambiguous value, $\Delta E_{\mathrm{D}}{ }^{\prime 26}{ }^{26}$ Unfortunately, despite its theoretical adequacy, this is impractical since exact densities are mostly obscure: Experimental electron densities from diffraction do not have the accuracy required, although this could change in the future for small systems. Furthermore, "exact" densities from quantum chemistry are beyond reach for almost all systems. Even for the closed-shell $1 \mathrm{~s}^{2}$ and $1 \mathrm{~s}^{2} 2 \mathrm{~s}^{2}$ systems studied by Medvedev et al., ${ }^{15}$ a high-level quantum chemical density may not be "exact," since errors mainly evaluated at the nucleus will be affected by erroneous nuclear cusps of most quantum-chemical methods that use Gaussian basis functions, unless designed to prevent this.

To solve this issue, we suggested using density sensitivity analysis by replacing the exact density with one or more densities of distinct known functionals. ${ }^{16}$ This simple protocol measures the global effect of variations in densities by computing $\mathrm{E}^{\prime \prime}\left[\rho^{\prime}\right]-\mathrm{E}^{\prime \prime}\left[\rho^{\prime \prime}\right]$, where $\rho^{\prime \prime}$ is the studied functional's self-consistent converged density, $E^{\prime \prime}$ is an energy evaluation with the functional, and $\rho^{\prime}$ is a converged Hohenberg-Kohn trial density [bottom-left cycle in Fig. $1(\mathrm{a})]$ rather than the elusive $\mathrm{E}\left[\rho^{\prime}\right]-\mathrm{E}\left[\rho_{\text {exact }}\right]$ [top-right cycle of Fig. 1(a)]. ${ }^{16}$ This protocol, easily applied in programs such as Turbomole, is closely related to the Hohenberg-Kohn variational principle, which determines the signs of the processes in Fig. 1(a). ${ }^{16}$ We recommend using two distinct theories to produce the densities such as a hybrid GGA (e.g., PBE0) and a local density approximation (LDA). $\Delta E_{\mathrm{D}}{ }^{\prime}$ is often small ("normal systems") since the functional's treatment of $\rho$ commonly matters more than the finer details of $\rho$ itself. This is the case for the study by Medvedev et al. once the energy impact of the reported density errors is evaluated but also for chemically relevant systems. ${ }^{16,25}$ Thus, the interesting question is not whether there are density errors but whether they are chemically significant. $^{16,26-28}$

To move forward on how to obtain energy-density balanced DFT, we note that in any chemical process, electrons are moved from one place to another such that the chemical potential is equal everywhere in a molecular system at equilibrium. ${ }^{24,29}$ The electronegativity $\chi$ is defined as the negative of the chemical potential at constant external potential $v(\mathrm{r})$, as seen in Fig. 1(b),

$$
\chi=-(\partial \mathrm{E} / \partial \mathrm{N})_{v(\mathrm{r})} \sim(\mathrm{IP}+\mathrm{EA}) / 2 .
$$

This expression with the ionization potential (IP) and electron affinity (EA) is the finite-difference approximation, i.e., Mulliken electronegativity. ${ }^{30}$ The hardness can be defined similarly as $3,29,31,32$

$$
\eta=\left(\partial \mathrm{E}^{2} / \partial \mathrm{N}^{2}\right)_{v(\mathrm{r})} \sim(\mathrm{IP}-\mathrm{EA}) .
$$

Since both the uptake and removal of electrons must be welldescribed at the equilibrium chemical potential and since these changes relate to the energy-density balance, we hypothesized that DFT may feature imbalanced chemical potentials. In the finitedifference approximation, this amounts to imbalances in $\chi=$ (IP + EA) $/ 2$ and $\eta=I P-E A$. Accordingly, DFT should display symmetry in terms of gaining and losing electrons and thus work well for the EA and IP for the same systems simultaneously, rather than for different systems as often tested. $\chi$ may then capture the
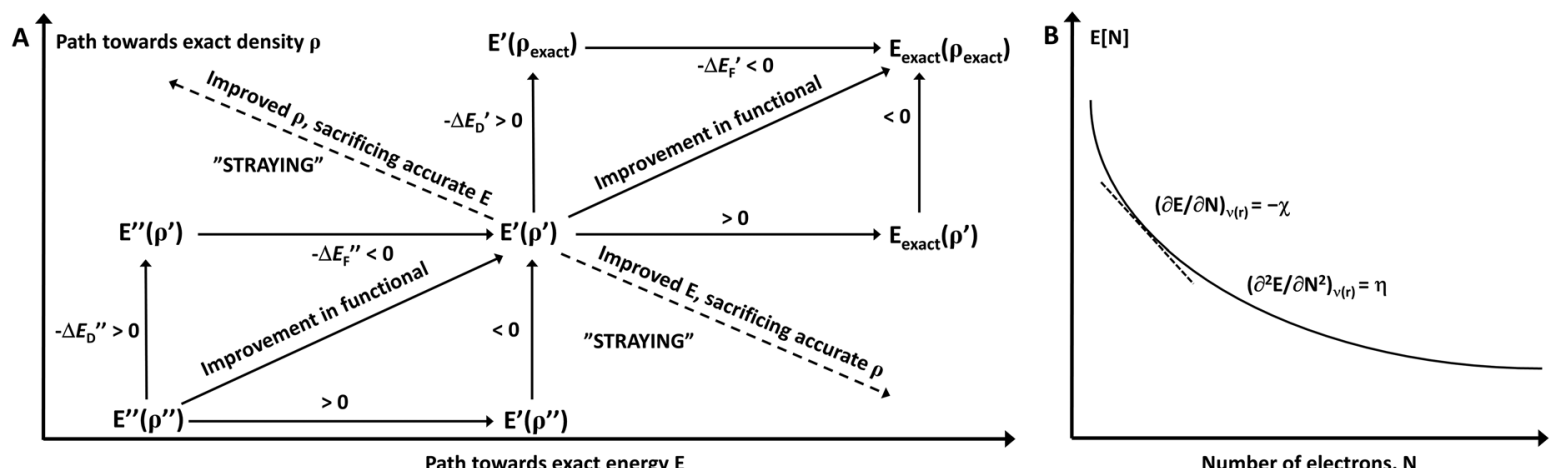

FIG. 1. Energy-density relationships, exactness, and electronegativity. (a) Energy-density relationships via trial functionals toward the exact functional based on Ref. 16 . The sign of each process follows from the Hohenberg-Kohn variational principle. ${ }^{10}$ (b) Definitions of electronegativity $\chi$ and hardness $\eta .{ }^{23}$ 
energy-density relationship that has been so debated recently. 15,16,25,28 Since $\chi$ excellently describes many types of reactivity and diverse chemical bond strengths ${ }^{33,34}$ and since the Pauling and Mulliken electronegativities correlate strongly, ${ }^{35}$ we propose that performance for $\chi$ is a "descriptor of universality" of interest to further development of DFT.

To explore this idea, we computed the hardness and electronegativity for all elements from $\mathrm{Z}=1-36$ for 50 diverse density functionals. Because of their importance, the atomic IPs and EAs have been studied massively with DFT. ${ }^{, 20,36-43}$ The absolute $\chi$ and $\eta$ for a range of molecules and four atoms were studied by Dixon and coworkers using several functionals. ${ }^{39}$ Hybrid DFT is somewhat less accurate for IPs and EAs than the G2 method but generally performs well for both on average. There has been confusion regarding DFT's ability to describe anions and thereby EAs. ${ }^{44,45}$ With large basis sets including diffuse functions, DFT is fully capable to do so in broad benchmarks. ${ }^{37,45}$ However, the required balance of IP and EA for the same systems is typically not obtained despite average good performance: For example, the IP of oxygen, a very important element, displays errors up to $0.4 \mathrm{eV}-1 \mathrm{eV}$, 37 which can only be remedied by special corrections. ${ }^{2}$

The novelty in the present work lies mainly in linking $\chi$ and $\eta$ to the energy-density balance, suggesting their use as probes of universality and offering a systematic study of 50 functionals, including both modern empirical and non-empirical density functionals, to test these ideas. We particularly emphasize the relationship between the accuracy and precision of DFT, which turns out to vary greatly when describing $\chi$ and $\eta$.

\section{METHODS}

\section{Energy computations and functionals studied}

We used the Gaussian $16^{46}$ software for all computations. IPs and EAs were computed for the 36 elements $\mathrm{Z}=1-36(\mathrm{H}-\mathrm{Kr})$. This choice was made because relativistic spin-orbit coupling affects the EA and IP of heavier atoms, and these are hard to evaluate accurately, both for energies ${ }^{47-49}$ but in particular their effect on densities. Our dataset covers both the s- and p-elements as well as the $3 \mathrm{~d}$ series, where major deviations from universality are expected to occur. ${ }^{50,51}$ Spin states for the atoms and atomic ions were taken from $\mathrm{NIST}^{52}$ and are summarized in Table S1. For a complete discussion of electron affinities, see the review by Rienstra-Kiracofe and coworkers. ${ }^{36}$ Experimental values for the IPs and EAs were taken from the CRC Handbook of Chemistry and Physics ${ }^{53}$ and are summarized in Table S2.

Energies were computed using the 50 functionals of Table I, with literature references, type, and amount of HF exchange (for the hybrid functionals) noted. These 50 functionals were chosen to (1) include many popular DFT functionals, ${ }^{54}$ (2) span many design types, and (3) include both older and newer functionals, as time has been claimed to work against universality because the energy is increasingly over-emphasized. ${ }^{15}$ We note that we use basis sets that provide chemical accuracy for $\operatorname{CCSD}(\mathrm{T}) .{ }^{16}$ The electron affinities depend on loosely bound anion states, which may be difficult to describe ${ }^{42,55,56}$ probably because of basis set limitations rather than failure of DFT itself. ${ }^{44,45}$ We used aug-cc-pV5Z ${ }^{57}$ except for
$\mathrm{K}$ and $\mathrm{Ca}$, which used def2-QZVPPD. ${ }^{58}$ All electronic energies are summarized in Tables S3-S11. The IPs were calculated as

$$
I P=E\left(X^{+}\right)-E(X) .
$$

$E(X)$ and $E\left(X^{+}\right)$are the single-point energies of neutral $X$ and its mono-cation $\mathrm{X}^{+}$. Correspondingly, the EAs were calculated as

$$
E A=-\left(E\left(X^{-}\right)-E(X)\right) .
$$

Here, $E\left(X^{-}\right)$is the single-point energy of the monoanion of element $\mathrm{X}$.

\section{Computing density-derived errors}

Burke's group has defined systems with large density-derived errors $\Delta E_{\mathrm{D}}{ }^{\prime}$ as "abnormal." ${ }^{26-28}$ A practical threshold for abnormality was suggested to be chemical accuracy, i.e., $4 \mathrm{~kJ} / \mathrm{mol} .{ }^{16}$ Burke has advocated $^{27,42}$ using a DFT non-consistent single-point energy calculation on the self-consistent HF density (HF-DFT) for abnormal electronic systems and found it to work well for EAs. ${ }^{42}$ Since pathological HF densities are common to systems with static correlation, as is in particular the case for the $3 \mathrm{~d}$-series, using HF densities may overestimate DFT abnormality. ${ }^{50}$ Instead, we follow the protocol of using density sensitivity analysis by testing how reasonable variations in density affect the total energy. ${ }^{16}$ As reasonable variations, we only consider DFT-derived densities, spanning from LDA to hybrid DFT, and favor non-empirical functionals.

We followed the protocol described previously. ${ }^{16}$ Specifically, we used PBE as a widely used non-empirical functional and evaluated the non-consistent PBE energy on the converged HF densities (referred to as HF-PBE), SVWN densities (SVWN-PBE), and PBE0 densities (PBE0-PBE) by setting the number of iterations for the SCF procedure to 1 and changing the density convergence threshold to $10^{7}$ a.u. in Turbomole. The obtained energies can be seen in Table S12. Sim et al. ${ }^{28}$ suggested a simple specific metric, $S\left(\mathrm{E}_{\mathrm{xc}}\right)$, for quantifying abnormality that involves only the non-consistent energies computed by PBE on the densities from HF and SVWN, ${ }^{99,105}$

$$
S\left(\mathrm{E}_{\mathrm{XC}}\right)=\mathrm{E}_{\mathrm{PBE}(\mathrm{SVWN})}-\mathrm{E}_{\mathrm{PBE}(\mathrm{HF})} .
$$

The first acronym in subscript represents the energy calculations and acronyms in parenthesis represent the methods used to compute the self-consistent densities. As an example, for EAs,

$$
\begin{aligned}
S^{\mathrm{EA}}{ }_{\mathrm{PBE}(\mathrm{SVWN}, \mathrm{HF})=} & \mathrm{E}_{\mathrm{PBE}(\mathrm{SVWN})}, \mathrm{X}^{-}-\mathrm{E}_{\mathrm{PBE}(\mathrm{HF})}, \mathrm{X}^{-} \\
& -\mathrm{E}_{\mathrm{PBE}(\mathrm{SVWN})}, \mathrm{X}+\mathrm{E}_{\mathrm{PBE}(\mathrm{HF})}, \mathrm{X},
\end{aligned}
$$

which can also be written as

$$
S_{\mathrm{PBE}(\mathrm{SVWN}, \mathrm{HF})}^{\mathrm{EA}}=\mathrm{EA}_{\mathrm{PBE}(\mathrm{SVWN})}-\mathrm{EA}_{\mathrm{PBE}(\mathrm{HF})} .
$$

Since HF densities may overestimate density sensitivities, we use our preferred metric,

$$
S_{\mathrm{PBE}(\mathrm{SVWN}, \mathrm{PBE} 0)}^{\mathrm{EA}}=\mathrm{EA} \mathrm{PBE}_{(\mathrm{SVWN})}-\mathrm{EA}_{\mathrm{PBE}(\mathrm{PBE} 0)} .
$$

These values applied to both IPs and EAs are summarized in Tables S13 and S14. 
TABLE I. Overview of the 50 exchange-correlation functionals studied in this work.

\begin{tabular}{|c|c|c|c|}
\hline Functional & Type & \% HF exchange & References \\
\hline APFD & Hybrid GGA & 23 & 59 \\
\hline B1B95 & Hybrid GGA & 28 & 60 \\
\hline B2PLYP & Double hybrid & 53 & 61 \\
\hline B3LYP & Hybrid GGA & 20 & $62-64$ \\
\hline B3P86 & Hybrid GGA & 20 & 65 and 66 \\
\hline B97-1 & Hybrid GGA & 21 & 67 \\
\hline B97-2 & Hybrid GGA & 21 & 68 \\
\hline B97-D & GGA & 0 & 69 \\
\hline B98 & Hybrid GGA & 22 & 70 \\
\hline BHandH & Hybrid GGA & 50 & 71 \\
\hline BHandHLYP & Hybrid GGA & 50 & 71 \\
\hline BLYP & GGA & 0 & 65 and 66 \\
\hline BMK & Hybrid meta GGA & 42 & 72 \\
\hline BP86 & GGA & 0 & 65 and 66 \\
\hline CAM-B3LYP & Range-separated hybrid & $19-65$ & 73 \\
\hline G96PBE & GGA & 0 & 17,74 , and 75 \\
\hline HCTH407 & GGA & 0 & 67,76 , and 77 \\
\hline HSE06 & Range-separated hybrid & $0-25$ & $78-84$ \\
\hline LC-wHPBE & Range-separated hybrid & $0-100$ & 80 and $85-87$ \\
\hline M06 & Hybrid meta GGA & 27 & 88 \\
\hline M06-L & Meta GGA & 0 & 89 \\
\hline M11 & Range-separated meta hybrid & $43-100$ & 90 \\
\hline M11-L & Meta GGA & 0 & 90 \\
\hline MN15 & Hybrid meta NGA & 44 & 91 \\
\hline MN15-L & Meta NGA & 0 & 92 \\
\hline mPW1PW91 & Hybrid GGA & 25 & $93-95$ \\
\hline mPW3PBE & Hybrid GGA & 25 & 17 and 93 \\
\hline N12-SX & Screened exchange NGA & & 96 \\
\hline O3LYP & Hybrid GGA & 12 & 97 \\
\hline OLYP & GGA & 0 & 63 and 98 \\
\hline OP86 & GGA & 0 & 66 and 98 \\
\hline OPBE & GGA & 0 & 17 and 98 \\
\hline OVWN & GGA & 0 & 98 and 99 \\
\hline PBE & GGA & 0 & 17 \\
\hline PBE0 & Hybrid GGA & 25 & 17 and 100 \\
\hline PW6B95 & Hybrid meta GGA & 28 & 101 \\
\hline PW6B95D3 & Hybrid meta GGA & 28 & 101 \\
\hline RevPBE0 & Hybrid GGA & 25 & 100 and 102 \\
\hline RevTPSS & Meta GGA & 0 & 103 \\
\hline RPBE & GGA & 0 & 104 \\
\hline SLYP & GGA & 0 & 65 and 105 \\
\hline SVWN & LSDA & 0 & 99 and 105 \\
\hline SVWN5 & LSDA & 0 & 99 and 105 \\
\hline tHCTH & Meta GGA & 0 & 106 \\
\hline tHCTHhyb & Hybrid meta GGA & 15 & 106 \\
\hline TPSS & Meta GGA & 0 & 107 \\
\hline TPSSh & Hybrid meta GGA & 10 & 107 \\
\hline VSXC & Meta GGA & 0 & 102 \\
\hline wB97XD & Range-separated hybrid & $22-100$ & 108 \\
\hline wPBEhPBE & GGA & 0 & $17,79,80$, and 109 \\
\hline X3LYP & Hybrid GGA & 22 & 96 \\
\hline
\end{tabular}




\section{RESULTS AND DISCUSSION}

\section{Hypothesis: Using $\chi=-\partial \mathrm{E} / \partial \mathrm{N}$ to probe universality}

The main hypothesis of the present work is that the chemical potential $\partial \mathrm{E} / \partial \mathrm{N}$ may not be balanced in most density functionals due to an over-emphasis on energies of neutral and cationic systems (enthalpies of formation and ionization energies), rather than densities of electron-rich systems. If so, the gain of electrons will be less well described than the loss of electrons. The performance for anions is sometimes challenged, ${ }^{44,55}$ and diffuse densities of anions, which decay exponentially, may be abnormal when the $1 / \mathrm{r}$ dependence of the exchange-correlation functional is violated (i.e., contribute large density-derived errors to the result). ${ }^{27,42,55}$ However, DFT performance for anions in broader benchmarks is generally fairly good, as measured by EAs, ${ }^{36,37,90}$ although cancellation of errors in the density and the functional [i.e., $\Delta E_{\mathrm{D}}{ }^{\prime}$ and $\Delta E_{\mathrm{F}}{ }^{\prime}$ of Fig. $1(\mathrm{a})$ ] possibly obscure the problem. Since we cannot generally measure the errors in the densities, using density sensitivity analysis is a possible alternative, ${ }^{16,28}$ as applied below, revealing large density errors in the $\mathrm{p}$ and d-blocks.

However, even with cancellation of errors in energy and density, systems may experience energy-density imbalance in terms of adding or removing electrons near the chemical potential $\partial \mathrm{E} / \partial \mathrm{N}$ [Fig. 1(b)] and its variation with $\mathrm{N}, \partial \mathrm{E}^{2} / \partial \mathrm{N}^{2}$, simply because the $\mathrm{X}, \mathrm{X}^{+}$, and $\mathrm{X}^{-}$systems were not considered together when developing DFT. Since the finite difference approximations to $\partial \mathrm{E} / \partial \mathrm{N}$ and $\partial \mathrm{E}^{2} / \partial \mathrm{N}^{2}$ are $-\chi$ and $\eta,{ }^{24,110}$ we assessed errors in $\chi$ and $\eta$ directly vs experimental data. An inspiration was that DFT errors tend to scale with effective nuclear charge, i.e., functionals are usually more accurate for either the left or right side of both the pand d-blocks. ${ }^{51,111}$ Since $\chi$ scales with effective nuclear charge, this may imply an imbalance in the chemical potential, $\partial \mathrm{E} / \partial \mathrm{N}$. Different functionals are accurate for different bonds types and electronic configurations, ${ }^{21,50,51,112}$ and errors from d-orbital occupation and spin scatter massively ${ }^{113-118}$ even for processes involving only a few atoms. ${ }^{118}$ We thus studied a significant part of the periodic table ( $\mathrm{Z}=1-36)$ to cover s-, $\mathrm{p}-$, and d-blocks while avoiding artifacts of relativistic energy.

\section{Distinct performance of $\mathbf{5 0}$ functionals for electronegativity and hardness}

Figure 2 summarizes the mean absolute errors (MAE) and mean signed errors (MSE) in the computed $\chi$ [Figs. 2(a)] and $\eta$ [Fig. 2(b)] of the 36 atoms, for the 50 studied density functionals, ranked according to MAE. We note that all values are without division of IP + EA by 2 to compare $\chi$ and $\eta$ more fairly. LDA performs much worse for $\chi$ than for $\eta$ [MAEs of $1.41 \mathrm{eV}$ and $1.52 \mathrm{eV}$ for SVWN and OVWN not shown due to scale in Fig. 2(a)], which we interpret as a major cancellation of errors in $\eta$, as EA is subtracted from the IP. Although uninteresting in terms of performance, the LDA functionals illustrate this most clearly. The corresponding performance for the IPs and EAs is summarized in Fig. S1. The MSEs of both IPs and EAs are approximately normal-distributed around zero, although slightly skewed toward too large EAs (Fig. S2), which indicates that we have sampled the DFT world well for the problem at hand.
Similarly, even functionals that perform decently for both IP and EA for the same systems may show larger errors in $\chi$ due to error propagation, since the IP and EA are added when calculating $\chi$. For example, BMK is the best functional for $\eta$ but average for $\chi$ because the decent MAEs for IPs and EAs $(0.13 \mathrm{eV})$ carry systematic errors that add in $\chi$. Specifically, since $\eta=E\left(X^{+}\right)+E\left(X^{-}\right)$ $-2 \mathrm{E}(\mathrm{X})$ and $\chi=\mathrm{E}\left(\mathrm{X}^{+}\right)-\mathrm{E}\left(\mathrm{X}^{-}\right), \eta$ reflects a disproportionation reaction, i.e., two single-electron transfers, whereas $\chi$ reflects twoelectron transfer (the sum of the first and second ionization energy of the anion state). The error cancellation is therefore expected to be larger in $\eta$. Indeed, we see that many standard functionals plateau at a MAE of $\sim 0.2 \mathrm{eV}-0.3 \mathrm{eV}$ [Fig. 2(b)]. In contrast, DFT performance for $\chi$ varies considerably: Typical GGAs show errors from $0.25 \mathrm{eV}$ to $0.50 \mathrm{eV}$ for $\chi$, whereas some hybrids and meta functionals display lower MAEs all the way down to $\sim 0.1 \mathrm{eV}$. Similarly, the standard deviation of the errors (gray thin bars in Fig. 2) oscillates wildly for $\chi$ [Fig. 2(a)] but is approximately symmetric around zero for $\eta$ [Fig. 2(b)].

Thus, apparent good performance for IPs and EAs, as commonly tested, hides major error cancellations from treating most of the electronic structure in the same way, yet $\chi$ reveals these cancellations. Since $\chi \approx-\partial \mathrm{E} / \partial \mathrm{N}$, we relate this to a commonly poor description of the chemical potential, which again implies a weakness in the incremental energy-density relationship, i.e., change in energy with small changes in electron count at fixed external potential, or similarly the electron density within a confined part of the system assuming a fixed volume. As the derivative of the $\mathrm{E} / \mathrm{N}$ relationship in Fig. 1(b), it implies that DFT is unbalanced in terms of adding or subtracting electrons from the same systems. A simple way to solve this problem is to ensure that the systematic errors (not MAEs) are close to zero for both IPs and EAs of the same systems (not different systems, as commonly applied in benchmark and parameterization datasets) by minimizing errors in $\chi$, which is much more sensitive than EA and IP separately. Still, this only ensures that functional-derived errors are minimized, i.e., that $\Delta E_{\mathrm{F}}{ }^{\prime} \rightarrow 0$; it does not ensure that the density-derived errors $\Delta E_{\mathrm{D}}{ }^{\prime}$ are not propagated at the same time.

We note from Fig. 2 that a handful of functionals perform particularly well for both $\chi$ and $\eta$. Functionals that feature in top-10 for both properties are B98, B97-1, and PW6B95D3. Since the latter functional is corrected by empirical dispersion, it may be a matter of taste if it should be listed. In the top-15 of both, we only find one more functional, APFD. In the top-20 of both, we find O3LYP, TPSS, B97-2, wB97XD, X3LYP, revPBE0, with HSE06 (No. 5 for $\chi$ for No. 21 for $\eta$ ) and PBE0 (No. 10 for $\chi$ for No. 22 for $\eta$ ) not far behind.

\section{The chemical potential is poorly described in the d-block}

The analysis above considered the total performance across the $\mathrm{s}^{-}, \mathrm{p}-$, and d-elements of the first four periods of the periodic table. In order to know which part of the periodic table produces most of the errors in $\partial \mathrm{E} / \partial \mathrm{N}$ and $\partial \mathrm{E}^{2} / \partial \mathrm{N}^{2}$ as probed by $\chi$ and $\eta$, the performance vs experiment was separated into elements for all the studied atoms. The main errors for IPs and EAs are summarized in the supplementary material, Tables S15 and S16, respectively. Generally, trend prediction is extremely good for IPs for all functionals $\left(R^{2}>0.99\right)$, 


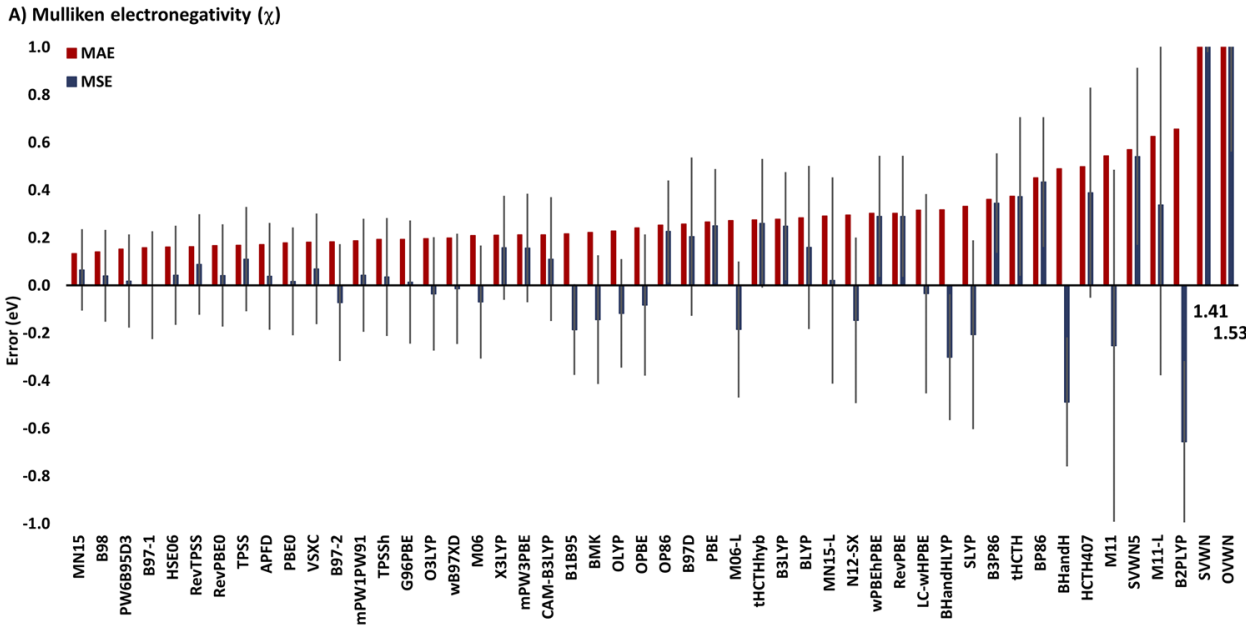

B) Absolute hardness ( $\eta$ )

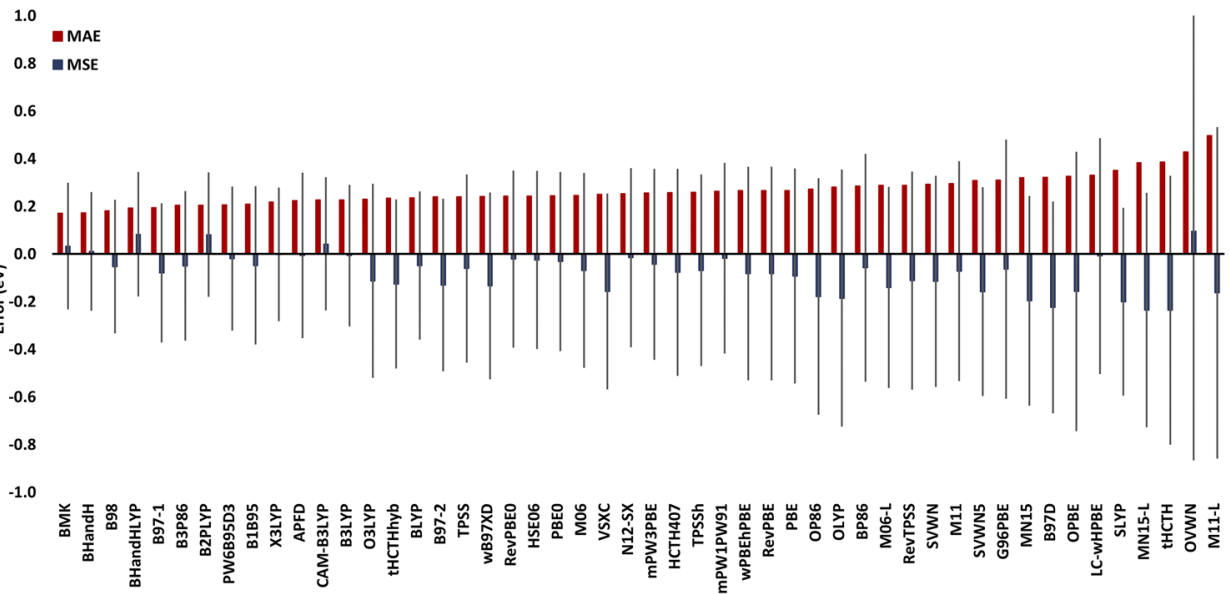

FIG. 2. Errors vs experiment (in eV) of 50 density functionals applied to atoms $Z=1-36$. The histograms show the MAEs (red bars), the MSEs (blue bars), and the standard deviations of the errors (thin gray bars). (a) Mulliken electronegativity $(\chi)$. (b) Absolute hardness ( $\eta$ ). In (a), the maxima of SVWN and OVWN (MAEs $=1.41 \mathrm{eV}$ and $1.52 \mathrm{eV}$ ) have been left out for better viewing and for putting the errors in $\chi$ and $\eta$ on the same scale. but this is largely due to the spread of energies across $25 \mathrm{eV}$, which obscures chemically significant errors. For EAs, which are numerically much smaller, trend prediction falls to typically $\mathrm{R}^{2} \sim 0.95$ and down to 0.7 for some LDA methods. Importantly, the full MAEs for IPs and EAs are largely comparable (Tables S15 or S16). Thus, DFT does not have a general problem with EAs over IPs when averaged over all 36 atoms, but this tendency hides certain very pathological cases.

To identify the main pathological systems, we use B98, one of the very best performing functionals, as example in Fig. 3. Other well-performing functionals such as B97-1 gave similar results (Fig. S3). Despite the excellent trend predictions, errors in the computed hardness for some $3 \mathrm{~d}$ metals [Fig. 3(a)] arise from corresponding errors in the EAs [Fig. 3(b)]. The pathology of the 3d series is also seen from the comparison of computed and experimental $\chi$ [Fig. 3(c)], in particular when zooming in on a smaller energy range [Fig. 3(d)]. From Table S15, where the maximum errors are listed for method, we see that the IPs of oxygen and boron are particularly pathological, confirming the old benchmark study by Curtiss and co-workers. ${ }^{37}$ The error for boron even for B98 is also evident from Fig. 3(d). For the typically very similarly performing GGA functionals BP86 and PBE and their derived methods, $\mathrm{Cr}$ is interestingly particularly pathological.

For the EAs, titanium is a major cause of error, followed by some other 3d-metals (Table S16). Since DFT across the board overestimates the EA of Ti and predicts it to trend with the EAs of Sc and V [Fig. 3(b)] as we might expect from the continued occupation of similar d-orbitals, one could question the experimental value $^{36}$ in this single case. However, considering the error's magnitude ( $\sim 0.5 \mathrm{eV}$ error for the best functionals), the possible uncertainty in this experimental value out of 36 does not change the performance reported above. Even excluding $\mathrm{Ti}$, the maximal EA errors of other functionals were exclusively seen for $3 \mathrm{~d}$ metals, except one case, MN11, which had largest errors for Br.

\section{Accuracy and precision of the chemical potential probed by $\chi$}

We have seen large differences in the description of $\eta$ and $\chi$ by DFT as evaluated by 50 distinct exchange-correlation functionals and found that functionals that are good for one property are rarely good for the other because $\eta$ cancels errors much better in 

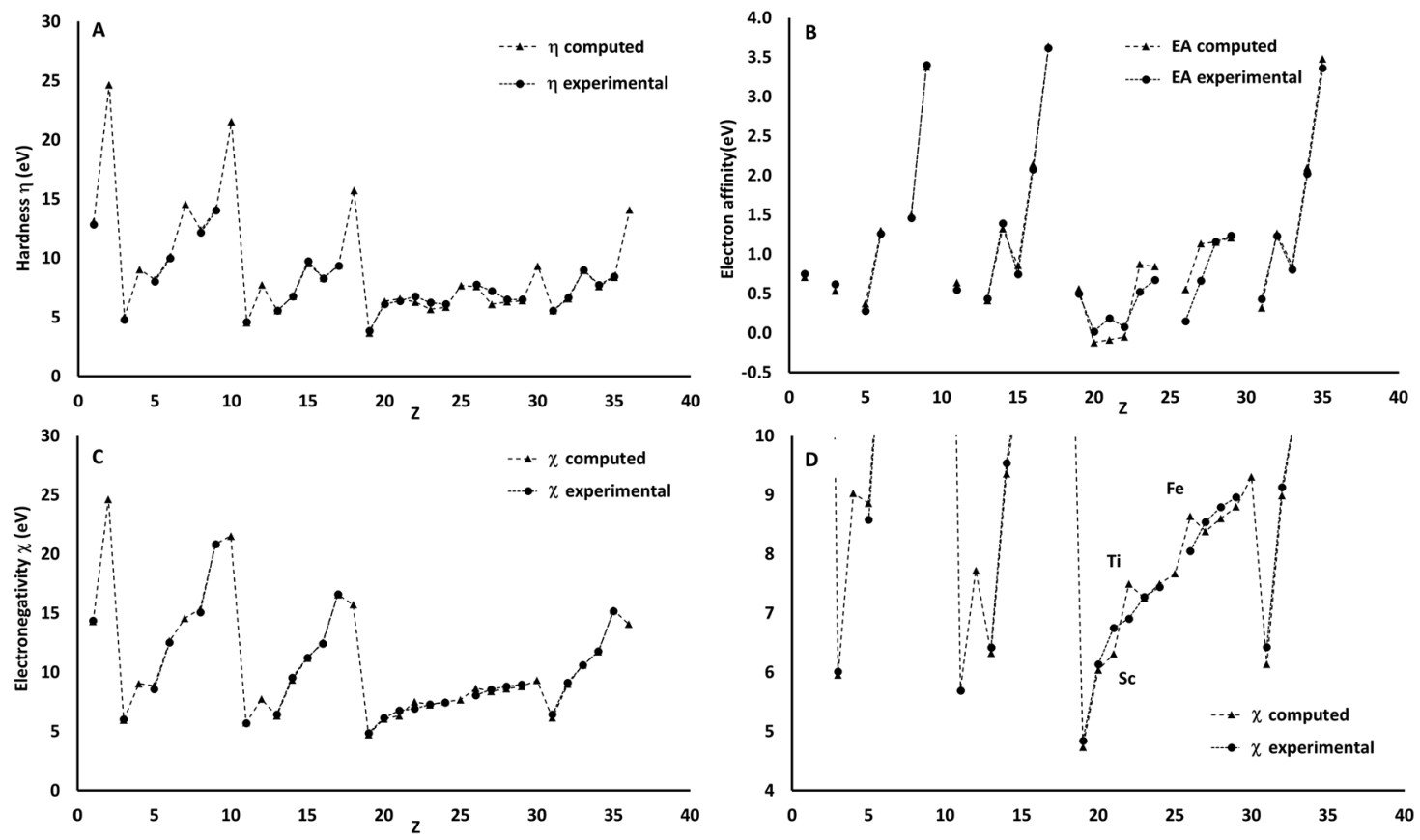

FIG. 3. Example of performance for one of the 50 studied functionals, B98. (a) Computed vs experimental $\eta$. (b) Computed vs experimental EAs. (c) Computed vs experimental $\chi$. (d) Computed vs experimental $\chi$ as in (c) but zoomed on values $<10 \mathrm{eV}$, with notable outliers marked. All values are in $\mathrm{eV}$.

the electronic structures of $\mathrm{X}, \mathrm{X}^{+}$, and $\mathrm{X}^{-}$. Most importantly, $\chi$, due to error propagation from the two-electron transfer process $\mathrm{E}^{+}-$ $\mathrm{E}^{-}$, when applied to the same system $\mathrm{X}$, is a sensitive probe of the description of the chemical potential, both with respect to loss and gain of electrons. Even the best performing functionals have problems with the gradual occupation of degenerate $\mathrm{p}$ - and d-orbitals. We also noted that the standard deviations of the errors are much more random for $\chi$ than for $\eta$, which could also imply that the precision differs from the accuracy of DFT applied to these simple processes.

To understand whether this is the case, Fig. 4 compares the accuracy, as measured by the MAE, to the systematic errors, measured by the MSE. Figure 4(a) shows the relationship for IPs, whereas Fig. 4(b) shows it for EAs, and Fig. 4(c) shows it for $\chi=I P+E A$. The MSE reflects the tendency to over-stabilize one of the states, either $\mathrm{X}, \mathrm{X}^{+}$, or $\mathrm{X}^{-}$(typically one of the two latter). We have suggested the use of these curves previously to pinpoint error relationships and room for improvement. The wellshapes or "inverse volcanos" represent the expectation that one would have if the MSEs were monotonously related to the MAEs. The reason for using curves rather than lines is that we expect a "hard" limit of accuracy to smooth out the bottom of the well, simply due to statistical noise. As seen from Figs. 4(a)-4(c), the hard limit of accuracy of $0.1 \mathrm{eV}$ is indeed seen in the bottom of the wells.

The IPs [Fig. 4(a)] show the largest deviation from the expected well shape because systematic errors are on average small and favor both $\mathrm{X}$ and $\mathrm{X}^{+}$, and thus their relationship with overall accuracy (MAE) is weaker, producing more scatter in Fig. 4(a). This tendency can also be seen from direct comparison of the methods in the histograms for IPs and EAs in Fig. S1. In contrast, the well shape is substantially more pronounced for the EAs [Fig. 4(b), please note the slight change of scale]. The majority of the 50 studied functionals have positive MSEs, i.e., they overestimate EA by favoring $\mathrm{X}^{-}$ relative to $\mathrm{X}$, which is particularly true for the local functionals (not shown; $0.63 \mathrm{eV}$ and $0.76 \mathrm{eV}$ MSE for SVWN and OVWN, Fig. S1) and GGA functionals such as PBE and BP86 $(\sim 0.17 \mathrm{eV}$ and $0.25 \mathrm{eV}$ MSE). This is also seen in the skewed distribution of errors (Fig. S2). Thus, anion states are in fact too stabilized when using sufficiently large basis sets with GGA DFT. For hybrid functionals, the balance is better, as reflected in the many functionals with small MSEs near the center of Fig. 4(b). Figure 4(c) shows the corresponding error plot for $\chi$; it is symmetric and follows the well shape, with the best functionals from the ranking in Fig. 2(a) also tending to have small systematic errors. For $\eta$, we found no well-shape but only a weak linear relationship (Fig. S4), again illustrating that systematic errors largely cancel for $\eta$.

Figures 4(d)-4(f) show the relationship between the accuracy, measured by the MAE, and the precision, measured by the standard deviation of the errors (gray bars in Fig. 2). The precision and accuracy of density functionals are not generally as strongly related as one could expect, as shown previously for spin-crossover systems. ${ }^{119}$ For the more generic IPs and EAs [Figs. 4(d) and 4(e)] and also for the computed $\chi$, we confirm this finding. The plots show a triangular shape with the highest accuracy (lowest MAE) being consistently associated also with higher precision. Functionals that stray from this relationship can be said to exhibit less predictable behavior, as the expected error becomes more uncertain. Thus, there are 

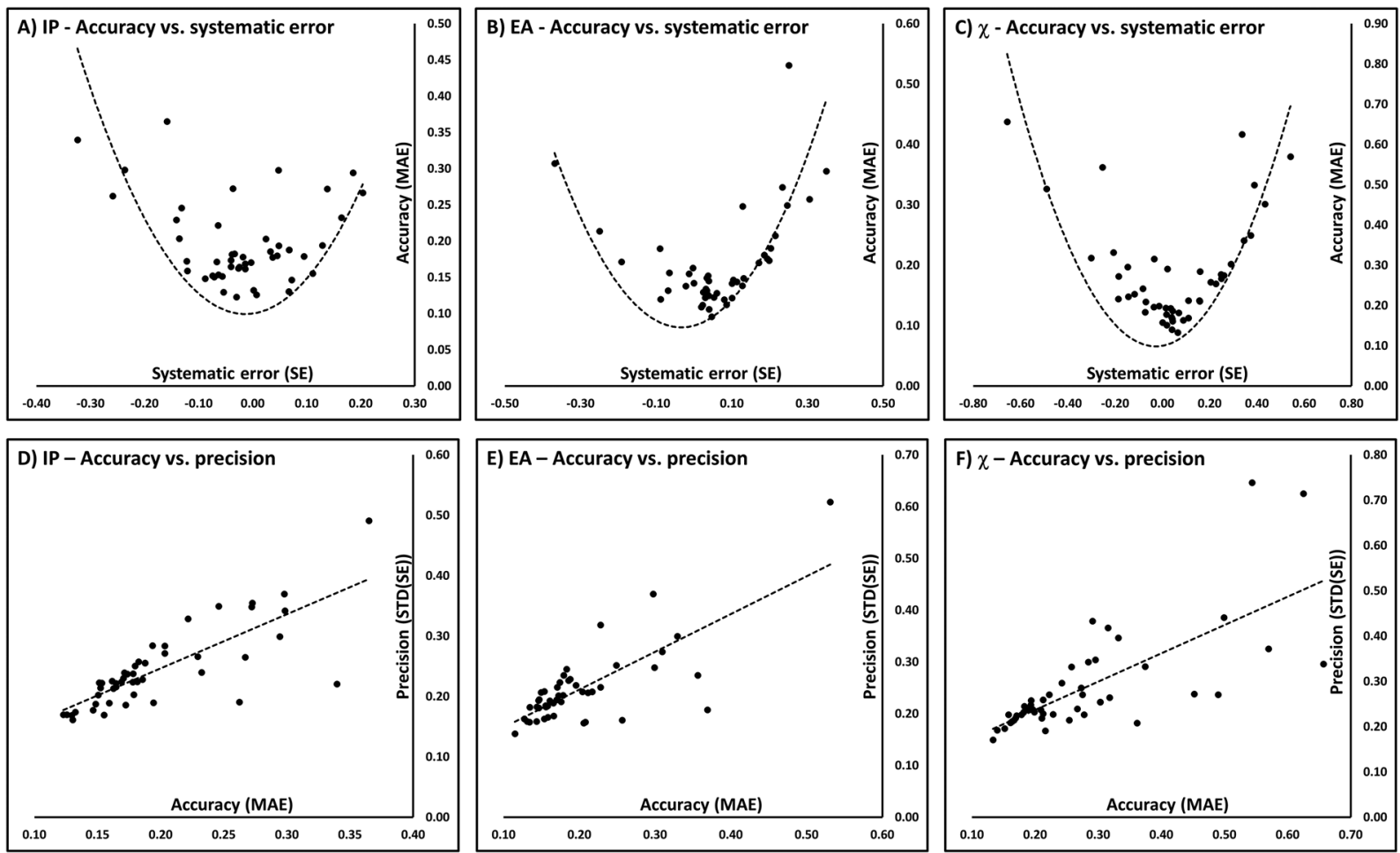

FIG. 4. Accuracy, precision, and systematic errors of density functionals (in eV). The LDA functionals OVWN and SVWN have been removed for better viewing. (a) Accuracy vs systematic errors (computed-experiment) for IPs. (b) Accuracy vs systematic errors for EAs. (c) Accuracy vs systematic errors for $\chi$. (d) Accuracy vs precision (standard deviations of the errors) for IPs. (e) Accuracy vs precision for EAs. (f) Accuracy vs precision for $\chi$.

functionals that are quite accurate but not very precise [on the lower side of the line in Figs. 4(d)-4(f)], while there are also functionals that are not so precise but of decent accuracy. We think these types of curves can be important as they may indicate potential overfitting of functionals in energy space, and for balanced nearly universal functionals, we expect a linear relationship to be fulfilled. The individual cases can be deduced from Tables S15 and S16, but for example, $\mathrm{BHandH}$ is more precise $(0.19 \mathrm{eV})$ than accurate $(0.26 \mathrm{eV})$ for EAs, whereas $\mathrm{MN} 15-\mathrm{L}$ is more imprecise $(0.43 \mathrm{eV})$ than it is accurate $(0.30 \mathrm{eV})$.

$X$ is the primary interest of this work, both via its excellent predictive power in broad chemistry, ${ }^{33}$ its close relationship to the theory via the chemical potential, ${ }^{24}$ and as a sensitive probe of imbalances in the energy-density relationship. Accordingly, the accuracyprecision relationship for this property [Fig. 4(f)] was clarified in more detail, with notable outliers shown in Fig. 5(a) and a zoomedin view on the best-performing functionals in Fig. 5(b). Even for these, the triangular shape is maintained. We note that $\mathrm{MN} 15^{91}$ performs excellently for $\chi$. This functional is the most broadly accurate of the Minnesota class and was parameterized to a very diverse range of data that included both IPs and EAs, explaining its success here. It demonstrates how far one can go with careful parameterization, yet all other functionals in the lower green quadrant of Fig. 5(b) represent nearly "non-empirical" functionals with very few parameters.
We also note that MN15 performs markedly worse for $\eta$ by error propagation, i.e., it has a negative MSE for IPs (Table S15) but a positive MSE for EA (Table S16), and these systematic errors add in $\eta$ to make $\partial \mathrm{E}^{2} / \partial \mathrm{N}^{2}$ poorly described, if this property is probed by $\eta$ as proposed by Parr and Pearson ${ }^{29}$ [Fig. 2(b)].

Thus, we emphasize that "universality" requires both $\chi$ and $\eta$ to be well-described for the same systems broadly. If doing so, as not done yet, since the chemical potential describes changing electron densities universally as reflected in the Pauling electronegativity, we hope that DFT can become more universal. In support of this assumption, we observe that the best-performing functionals across both properties, B98, PW6B95D3, and B97-1, have been recently shown to be particularly accurate in detailed benchmarks of very diverse metal-ligand bonds. ${ }^{50,51,120}$ Accordingly, a balanced description of $\partial \mathrm{E} / \partial \mathrm{N}$ and $\partial \mathrm{E}^{2} / \partial \mathrm{N}^{2}$, via $\chi$ and $\eta$, is an important test descriptor of DFT and probably a first simple probe of DFT universality.

\section{Contribution of densities to errors in the chemical potential probed by $x$}

As the last question of interest, we wanted to understand whether the errors observed are largely reflected by the energyfunctional space or whether the densities contribute to some of the 

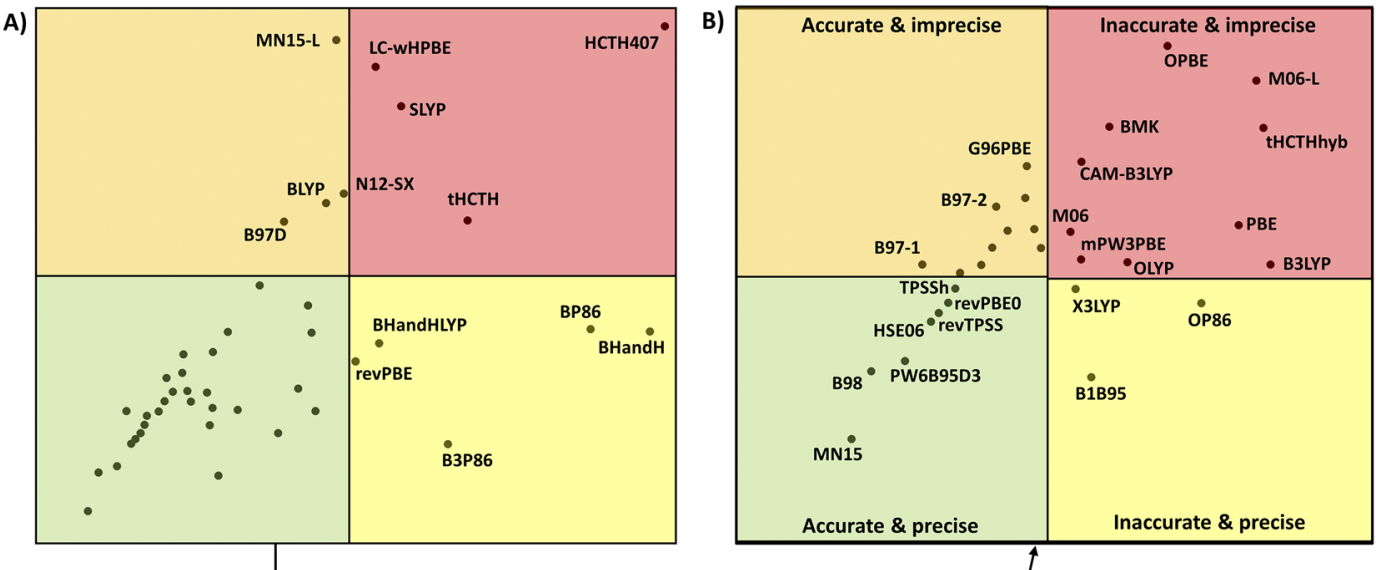

FIG. 5. Clustering of density functionals in accuracy-precision space. The plot is quantified by MAE (horizontal axis) and standard deviation of errors (vertical axis): (a) Cutoff: $0.5 \mathrm{eV}$ (i.e., the two local DFT methods SVWN and OVWN were excluded). (b) Cutoff: $0.3 \mathrm{eV}$.

pathology, as claimed in some cases for anion states, which can be described better in some cases by HF densities. ${ }^{27,55}$ Since the exact densities corresponding to the experimental IPs and EAs are unknown and since quantum chemistry cannot produce them accurately enough (except perhaps by full-CI using Slater orbitals to mimic the nuclear cusp), any comparison of densities is conditional, and we therefore proposed using density sensitivity analysis, ${ }^{16}$ as explained in the introduction. Here, we consider the two simplest metrics of abnormality [systems with large $\Delta E_{\mathrm{D}}{ }^{\prime}$ in Fig. 1(a)] that we consider most useful, the specific version suggested by Sim and co-workers ${ }^{28}$ that compares PBE on SVWN and HF densities and our version that compares PBE on SVWN and PBE0 densities. The advantage of these methods is their simplicity; in comparison to more elaborate metrics that average over more rungs of the DFT ladder, ${ }^{16}$ they tend to maximize estimates of abnormality since the applied theories to compute the densities are quite diverse.

Figure 6 compares the values of these two metrics for the IPs [Fig. 6(a)], EAs [Figs. 6(b)], and X [Fig. 6(c)]. For the IPs, we see that the HF-based metric $S^{\mathrm{IP}}{ }_{\mathrm{PBE}(\mathrm{SVwN}, \mathrm{HF})}$ suggested by Burke, ${ }^{28}$ Eq. (7), is always larger than our PBE0-based metric, ${ }^{16}$ Eq. (8), as expected because it represents a large difference in applied theory, with HF heavily favoring loose densities and weaker binding, whereas LDA strongly overbinds.

For the IPs [Fig. 6(a)], the two metrics are in qualitative agreement, and both confirm that the $3 \mathrm{~d}$-series is extremely densitysensitive, with effects as large as $1 \mathrm{eV}(100 \mathrm{~kJ} / \mathrm{mol})$ most notably for $\mathrm{Co}$ and $\mathrm{Fe}$, the first clear data showing this for the $3 \mathrm{~d}$ series. The p-block is also sensitive but only up to $0.1 \mathrm{eV}(10 \mathrm{~kJ} / \mathrm{mol})$, and when using our DFT-based metric, the effects are negligible. The d-block harbors large density sensitivity partly due to distinct electronic configurations of the HF and DFT treatment, with the $4 \mathrm{~s}-3 \mathrm{~d}$ energetics being a main difficulty. We tried to optimize PBE and $\mathrm{HF}$ in alternative configurations to test the electronic configuration dependence but failed in both cases, indicating that the "excited" configurations are too unstable in both theories. For the DFT-based metric, the sensitivity at the early d-block mostly disappears because the theories converge to the same configurations. Since DFT is a ground state theory, we must accept the change in lowest energy configuration, which arises from the near-degeneracy and static correlation, which makes DFT and HF very different in the $3 \mathrm{~d}$ series.

For the EAs [Fig. 6(b)], which may be more abnormal (i.e., displaying larger density-derived errors) and sometimes improved by using HF densities, ${ }^{42,55}$ we indeed see more density sensitivity. Surprisingly, the HF metric predicts extreme density sensitivity for the $2 \mathrm{p}$-series (up to $0.8 \mathrm{eV}$ ) probably due to the static correlation of nearly degenerate p-orbitals. Confirming this interpretation, s-block elements, where degenerate occupation issues are absent, are unproblematic in all cases for both metrics. The effect only emerges in the anion state, not the neutral and cation state, thus confirming earlier work. ${ }^{42,55}$ Importantly, although both metrics indicate that the d-block produces large density-derived errors, they disagree on the p-block and the affected $3 \mathrm{~d}$ metals, i.e., the metrics give different results, even qualitatively, for EAs. As discussed previously, ${ }^{16,50}$ we do not believe that HF theory estimates density errors well due to its pathologies relative to DFT, as largely confirmed by Fig. 6. Thus, we still favor the metric of Eq. (8), ${ }^{50}$ shown in orange color, for estimating density sensitivity of DFT. For the same reason, HF-DFT (where HF densities are used with DFT energies) may work mainly in cases of error cancellation. The PBE0 and SVWN theories are almost maximally different within DFT and favor loose and tight binding, respectively, yet do not suffer the pathology that causes large effects in HF densities.

In terms of EAs, we note that the tails of the exchangecorrelation potentials do not generally obey the $1 / \mathrm{r}$ dependence, and thus, assessing the impact of this violation is of interest. To do so, we reanalyzed the EAs of the range-separated functionals wB97XD (22\%-100\%), M11 (43\%-100\%), LC-wHPBE (0\%-100\%), and CAMB3LYP (19\%-65\%), which were designed to account for both limits of the electron density. These functionals, marked blue in Table S16, only perform marginally better for EAs than 

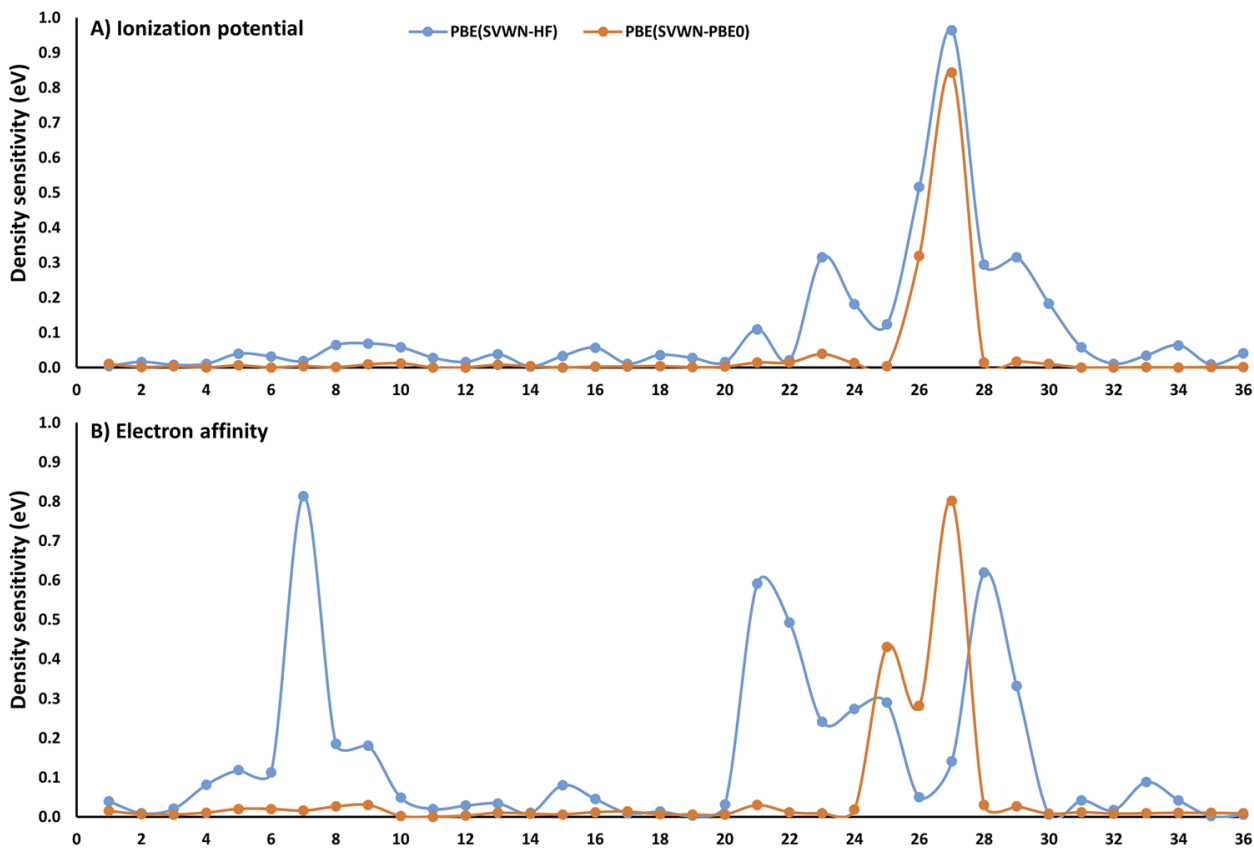

FIG. 6. Density sensitivities calculated using PBE on SVWN, HF, and PBEO densities. (a) lonization potentials for all atoms $Z=1-36$. (b) Electron affinities for $Z=1-36$. (c) Electronegativities for $Z=1-36$. All values are in $\mathrm{eV}$ and describe how much a change in density from HF to SVWN or from PBE0 to SVWN affects the PBE-computed property.

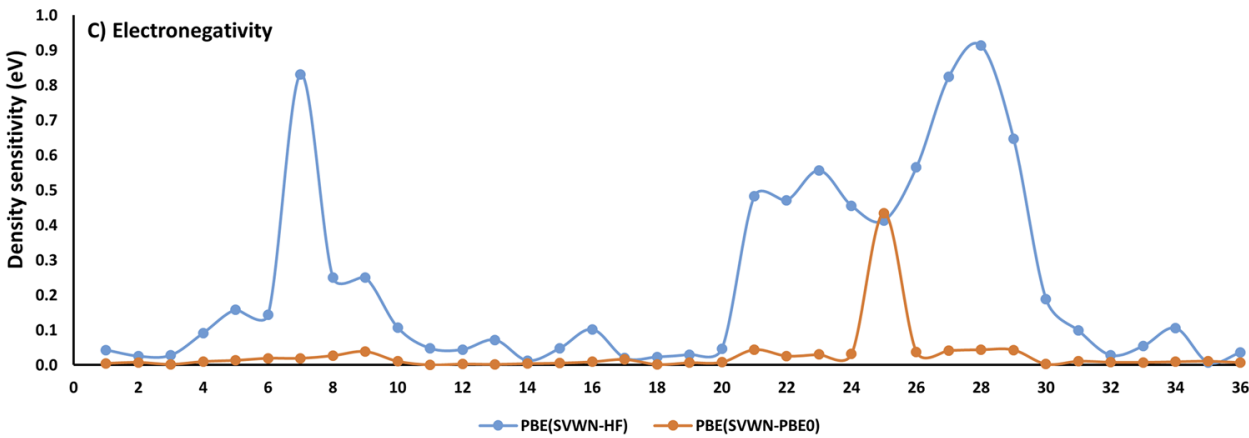

average, when excluding the LDA functionals (averages at the bottom of Table S16). This is consistent with benchmarks ${ }^{36,37,90}$ showing decent performance of functionals with moderate HF exchange and probably reflects that, although the densities are very pathological at the tail, the electron energies are very small far from the nucleus, thus making the effect on EAs (but not necessarily other energies such as transition state barriers) somewhat smaller than one would initially suspect.

Finally, the density sensitivity for the most important property studied in this work, the finite-difference approximation to the chemical potential- $\partial \mathrm{E} / \partial \mathrm{N}$ (i.e., $\chi$ ), is shown in Fig. 6 (c). Remarkably, while the HF metric suggests that density plays a major role in the errors across the p- and d-blocks (blue), with errors again reaching $1 \mathrm{eV}$, correlated DFT features much more efficient cancellation of errors of having mostly similar orbitals occupied in the $\mathrm{X}^{+}$and $\mathrm{X}^{-}$ states, and thus, the density sensitivity is much smaller for $\partial \mathrm{E} / \partial \mathrm{N}$ for the DFT-based than for the HF-based metric, consistent with the expectation that errors in the energy-density relationship cancel in the finite difference $\mathrm{E}\left(\mathrm{X}^{+}\right)-\mathrm{E}\left(\mathrm{X}^{-}\right)$when correlation effects of the "double electron transfer" are well accounted for. The only exception is seen for $\mathrm{Mn}$ in the middle of the d-block, which has a heptet $4 s^{1} 3 d^{5}$ cation state, a neutral $4 s^{2} 3 d^{5}$ state, and is the only state not showing having error cancellation in the DFT metric.

In summary, although the configuration dependence complicates the picture, Fig. 6 clearly shows the density sensitivity of the $2 p$ series and $3 \mathrm{~d}$ series as outstanding, and they coincide with the largest errors in the computed energies, as discussed above. We note that the kinetic energy contributes to the density errors. ${ }^{121}$ Large density gradients of the $\mathrm{p}$ - and d-elements related to the kinetic energy easily contribute to density sensitivity, and thus, errors are not only due to potential energy. We have not intended to divide the density-derived errors into potential and kinetic energy in the present work.

\section{CONCLUSIONS}

The quest toward universal functionals dates back to the original formulations by Hohenberg and Kohn. ${ }^{10}$ Transferability across 
the periodic table is lacking, as is commonly a balanced description of the electronic energy and density. ${ }^{15,25,27}$ There are two main approaches to this challenge: the use of extensively parameterized functionals ${ }^{19,20}$ and the use of fundamental bounds, which may aid universal behavior with few parameters. ${ }^{6,17}$ There are no simple metrics of universality to aid this quest, yet we expect that such metrics could help rationalizing and improving DFT results.

We hypothesized that the chemical potential, $\partial \mathrm{E} / \partial \mathrm{N}$, is not balanced in current DFT. $\partial \mathrm{E} / \partial \mathrm{N}$ is of major importance to chemical reactions: In any chemical process, electrons are moved from one place to another such that $\partial \mathrm{E} / \partial \mathrm{N}$ is equal in all parts of the molecular system at equilibrium. We hypothesized that DFT is unbalanced because both the uptake and removal of electrons need to be probed well around the chemical potential of the same systems, which has typically not been required during functional development. In the finite difference approximation, this amounts to performing well both for absolute electronegativities $\chi=I P+E A$ and hardness $\eta=I P$ - EA for the same systems specifically, rather than for IPs and EAs of different systems more broadly.

We have studied how 50 different density functionals describe $\chi$ and $\eta$. We find that (1) pathological cases are not due to anions per se but to adding electrons to degenerate $\mathrm{p}$ - and d-orbitals. (2) Popular functionals such as B3LYP, PBE, and revPBE perform poorly for both properties. (3) Functionals that are good for $X$ are commonly not good for $\eta$ and vice versa. For example, B3LYP, MN15, and MN15-L are quite good for IPs, but not for EAs, and therefore produce poor $\chi$ and $\eta$. (4) The accuracy and precision of a functional is not generally linearly related, yet for a universal functional, we expect linearity, and the best-performing functionals support this notion. Deviations from the accuracy-precision relationship are often seen for highly parameterized functionals. (5) The pathology of $\partial \mathrm{E} / \partial \mathrm{N}$ in the d-block as probed by $\chi$ is accompanied by large density-derived errors, as revealed by density sensitivity analysis.

Based on these results, we argue that balanced good performance for $\chi$ is a hallmark of universality by probing both sides of $\partial \mathrm{E} / \partial \mathrm{N}$. With this metric, B98, B97-1, PW6B95D3, APFD are the most "universal" among the tested functionals. In previous work, B98 and B97-1, and to some extent PW6B95D3, excellently described very diverse metal-ligand bonds across $3 \mathrm{~d}-$-, $4 \mathrm{~d}-$, and $5 \mathrm{~d}$-metals and ligands such as oxide, halides, and hydride. ${ }^{50,51,120}$ This seems to support that a balanced description of $\partial \mathrm{E} / \partial \mathrm{N}$ and $\partial \mathrm{E}^{2} / \partial \mathrm{N}^{2}$, via $\chi$ and $\eta$, is important to chemistry broadly and thus a very simple feature of universality that requires only the calculation of IP + EA for the same system, compared to experiment.

\section{SUPPLEMENTARY MATERIAL}

See the supplementary material for all the computed electronic energies and details of the analysis, including the density sensitivity metrics.

\section{ACKNOWLEDGMENTS}

We kindly acknowledge the use of the High-Performance Computing Cluster at DTU for the computations carried out in this work.
There is no funding to be acknowledged in this study.

\section{DATA AVAILABILITY}

The data that support the findings of this study are available from the corresponding author upon reasonable request.

\section{REFERENCES}

${ }^{1}$ W. Kohn, A. D. Becke, and R. G. Parr, J. Phys. Chem. 100, 12974 (1996).

${ }^{2}$ A. D. Becke, J. Chem. Phys. 140, 18A301 (2014).

${ }^{3}$ R. G. Parr, in Horizons Quantum Chemistry (Springer, 1980), pp. 5-15.

${ }^{4}$ K. P. Kepp, Coord. Chem. Rev. 257, 196 (2013).

${ }^{5}$ S. Lehtola, Int. J. Quantum Chem. 119, e25968 (2019).

${ }^{6}$ J. Tao, J. P. Perdew, V. N. Staroverov, and G. E. Scuseria, Phys. Rev. Lett. 91, 146401 (2003).

${ }^{7}$ T. Schwabe, Phys. Chem. Chem. Phys. 16, 14559 (2014)

${ }^{8}$ A. J. Cohen, P. Mori-Sánchez, and W. Yang, Chem. Rev. 112, 289 (2011).

${ }^{9}$ X.-M. Tong and S.-I. Chu, Phys. Rev. A 57, 855 (1998).

${ }^{10} \mathrm{P}$. Hohenberg and W. Kohn, Phys. Rev. 136, B864 (1964).

${ }^{11}$ M. Levy, Proc. Natl. Acad. Sci. U. S. A. 76, 6062 (1979).

${ }^{12}$ R. Peverati and D. G. Truhlar, Philos. Trans. R. Soc., A 372, 20120476 (2014).

${ }^{13}$ J. C. Slater, J. Chem. Phys. 57, 2389 (1972).

${ }^{14}$ M. Levy and J. P. Perdew, Phys. Rev. A 32, 2010 (1985).

${ }^{15}$ M. G. Medvedev, I. S. Bushmarinov, J. Sun, J. P. Perdew, and K. A. Lyssenko, Science 355, 49 (2017).

${ }^{16}$ K. P. Kepp, Phys. Chem. Chem. Phys. 20, 7538 (2018).

${ }^{17}$ J. P. Perdew, K. Burke, and M. Ernzerhof, Phys. Rev. Lett. 77, 3865 (1996).

${ }^{18}$ J. Sun, A. Ruzsinszky, and J. P. Perdew, Phys. Rev. Lett. 115, 036402 (2015).

${ }^{19}$ Y. Zhao and D. G. Truhlar, Chem. Phys. Lett. 502, 1 (2011).

${ }^{20}$ E. H. Knoll and R. A. Friesner, J. Phys. Chem. B 110, 18787 (2006).

${ }^{21}$ K. P. Jensen and U. Ryde, J. Phys. Chem. A 107, 7539 (2003).

${ }^{22}$ J. P. Perdew, AIP Conf. Proc. 577, 1 (2001).

${ }^{23}$ W. Yang and R. G. Parr, Proc. Natl. Acad. Sci. U. S. A. 82, 6723 (1985).

${ }^{24}$ R. G. Parr, R. A. Donnelly, M. Levy, and W. E. Palke, J. Chem. Phys. 68, 3801 (1978).

${ }^{25}$ K. P. Kepp, Science 356, 496 (2017).

${ }^{26}$ M.-C. Kim, E. Sim, and K. Burke, Phys. Rev. Lett. 111, 073003 (2013).

${ }^{27}$ A. Wasserman, J. Nafziger, K. Jiang, M.-C. Kim, E. Sim, and K. Burke, Annu. Rev. Phys. Chem. 68, 555 (2017).

${ }^{28}$ E. Sim, S. Song, and K. Burke, J. Phys. Chem. Lett. 9, 6385 (2018).

${ }^{29}$ R. G. Parr and R. G. Pearson, J. Am. Chem. Soc. 105, 7512 (1983).

${ }^{30}$ R. S. Mulliken, J. Chem. Phys. 2, 782 (1934).

${ }^{31}$ R. G. Pearson, J. Am. Chem. Soc. 107, 6801 (1985).

${ }^{32}$ R. G. Parr and J. L. Gazquez, J. Phys. Chem. 97, 3939 (1993).

${ }^{33}$ D. Bergmann and J. Hinze, Angew. Chem., Int. Ed. Engl. 35, 150 (1996).

${ }^{34}$ L. Pauling, J. Am. Chem. Soc. 54, 988 (1932).

${ }^{35}$ H. A. Skinner and H. O. Pritchard, Trans. Faraday Soc. 49, 1254 (1953).

${ }^{36}$ J. C. Rienstra-Kiracofe, G. S. Tschumper, H. F. Schaefer, S. Nandi, and G. B. Ellison, Chem. Rev. 102, 231 (2002).

${ }^{37}$ L. A. Curtiss, P. C. Redfern, K. Raghavachari, and J. A. Pople, J. Chem. Phys. 109, 42 (1998).

${ }^{38}$ D. J. Tozer and F. De Proft, J. Phys. Chem. A 109, 8923 (2005).

${ }^{39}$ C.-G. Zhan, J. A. Nichols, and D. A. Dixon, J. Phys. Chem. A 107, 4184 (2003).

${ }^{40}$ S. McKechnie, G. H. Booth, A. J. Cohen, and J. M. Cole, J. Chem. Phys. 142, 194114 (2015).

${ }^{41}$ Z. J. Wu and Y. Kawazoe, Chem. Phys. Lett. 423, 81 (2006).

${ }^{42}$ D. Lee, F. Furche, and K. Burke, J. Phys. Chem. Lett. 1, 2124 (2010).

${ }^{43}$ E. Kraisler, G. Makov, and I. Kelson, Phys. Rev. A 82, 042516 (2010).

${ }^{44}$ J. M. Galbraith and H. F. Schaefer III, J. Chem. Phys. 105, 862 (1996)

${ }^{45}$ N. Rösch and S. B. Trickey, J. Chem. Phys. 106, 8940 (1997). 
${ }^{46}$ M. J. Frisch, G. W. Trucks, H. B. Schlegel, G. E. Scuseria, M. A. Robb, J. R. Cheeseman, G. Scalmani, V. Barone, G. A. Petersson, H. Nakatsuji, X. Li, M. Caricato, A. V. Marenich, J. Bloino, B. G. Janesko, R. Gomperts, B. Mennucci, H. P. Hratchian, J. V. Ortiz, A. F. Izmaylov, J. L. Sonnenberg, D. Williams-Young, F. Ding, F. Lipparini, F. Egidi, J. Goings, B. Peng, A. Petrone, T. Henderson, D. Ranasinghe, V. G. Zakrzewski, J. Gao, N. Rega, G. Zheng, W. Liang, M. Hada, M. Ehara, K. Toyota, R. Fukuda, J. Hasegawa, M. Ishida, T. Nakajima, Y. Honda, O. Kitao, H. Nakai, T. Vreven, K. Throssell, J. A. Montgomery, Jr., J. E. Peralta, F. Ogliaro, M. J. Bearpark, J. J. Heyd, E. N. Brothers, K. N. Kudin, V. N. Staroverov, T. A. Keith, R. Kobayashi, J. Normand, K. Raghavachari, A. P. Rendell, J. C. Burant, S. S. Iyengar, J. Tomasi, M. Cossi, J. M. Millam, M. Klene, C. Adamo, R. Cammi, J. W. Ochterski, R. L. Martin, K. Morokuma, O. Farkas, J. B. Foresman, and D. J. Fox, Gaussian 16 rev., Gaussian Inc., Wallingford, CT, 2016.

${ }^{47}$ P. Pyykkö, Annu. Rev. Phys. Chem. 63, 45 (2012).

${ }^{48}$ M. K. Armbruster, F. Weigend, C. van Wüllen, and W. Klopper, Phys. Chem. Chem. Phys. 10, 1748 (2008).

${ }^{49}$ F. Neese, J. Chem. Phys. 118, 3939 (2003).

${ }^{50}$ K. A. Moltved and K. P. Kepp, J. Phys. Chem. A 123, 2888 (2019).

${ }^{51}$ K. A. Moltved and K. P. Kepp, J. Chem. Theory Comput. 14, 3479 (2018).

${ }^{52}$ A. Kramida, Y. Ralchenko, J. Reader, and NIST ASD Team, NIST Atomic Spectra Database (National Institute of Standards and Technology, Gaithersburg, MD, 2018), available at http://physics.nist.gov/asd.

${ }^{53} \mathrm{~J}$. Rumble, CRC Handbook of Chemistry and Physics, 98th ed. (CRC Press LLC, 2017).

${ }^{54}$ L. Goerigk and N. Mehta, Aust. J. Chem. 72, 563 (2019).

${ }^{55}$ M.-C. Kim, E. Sim, and K. Burke, J. Chem. Phys. 134, 171103 (2011).

${ }^{56}$ D. Lee and K. Burke, Mol. Phys. 108, 2687 (2010).

${ }^{57}$ T. H. Dunning, J. Chem. Phys. 90, 1007 (1989).

${ }^{58}$ F. Weigend and R. Ahlrichs, Phys. Chem. Chem. Phys. 7, 3297 (2005).

${ }^{59}$ A. Austin, G. A. Petersson, M. J. Frisch, F. J. Dobek, G. Scalmani, and K. Throssell, J. Chem. Theory Comput. 8, 4989 (2012).

${ }^{60}$ A. D. Becke, J. Chem. Phys. 104, 1040 (1996).

${ }^{61}$ S. Grimme, J. Chem. Phys. 124, 034108 (2006).

${ }^{62}$ P. J. Stephens, F. J. Devlin, C. F. Chabalowski, and M. J. Frisch, J. Phys. Chem. 98, 11623 (1994).

${ }^{63}$ C. Lee, W. Yang, and R. G. Parr, Phys. Rev. B 37, 785 (1988).

${ }^{64}$ A. D. Becke, J. Chem. Phys. 98, 5648 (1993).

${ }^{65}$ A. D. Becke, Phys. Rev. A 38, 3098 (1988).

${ }^{66}$ J. P. Perdew, Phys. Rev. B 33, 8822 (1986).

${ }^{67}$ F. A. Hamprecht, A. J. Cohen, D. J. Tozer, and N. C. Handy, J. Chem. Phys. 109, 6264 (1998).

${ }^{68}$ P. J. Wilson, T. J. Bradley, and D. J. Tozer, J. Chem. Phys. 115, 9233 (2001).

${ }^{69}$ S. Grimme, J. Comput. Chem. 27, 1787 (2006).

${ }^{70}$ H. L. Schmider and A. D. Becke, J. Chem. Phys. 108, 9624 (1998).

${ }^{71}$ A. D. Becke, J. Chem. Phys. 98, 1372 (1993).

${ }^{72}$ A. D. Boese and J. M. L. Martin, J. Chem. Phys. 121, 3405 (2004).

${ }^{73}$ T. Yanai, D. P. Tew, and N. C. Handy, Chem. Phys. Lett. 393, 51 (2004).

${ }^{74} \mathrm{C}$. Adamo and V. Barone, J. Comput. Chem. 19, 418 (1998).

${ }^{75}$ P. M. W. Gill, Mol. Phys. 89, 433 (1996).

${ }^{76}$ A. D. Boese and N. C. Handy, J. Chem. Phys. 114, 5497 (2001).

${ }^{77}$ A. D. Boese, N. L. Doltsinis, N. C. Handy, and M. Sprik, J. Chem. Phys. 112, 1670 (2000).

${ }^{78}$ A. V. Krukau, O. A. Vydrov, A. F. Izmaylov, and G. E. Scuseria, J. Chem. Phys. 125, 224106 (2006).

${ }^{79}$ A. F. Izmaylov, G. E. Scuseria, and M. J. Frisch, J. Chem. Phys. 125, 104103 (2006).
${ }^{80}$ T. M. Henderson, A. F. Izmaylov, G. Scalmani, and G. E. Scuseria, J. Chem. Phys. 131, 044108 (2009).

${ }^{81}$ J. Heyd, G. E. Scuseria, and M. Ernzerhof, J. Chem. Phys. 124, 219906 (2006).

${ }^{82}$ J. Heyd, J. E. Peralta, G. E. Scuseria, and R. L. Martin, J. Chem. Phys. 123, 174101 (2005).

${ }^{83}$ J. Heyd and G. E. Scuseria, J. Chem. Phys. 120, 7274 (2004).

${ }^{84}$ J. Heyd and G. E. Scuseria, J. Chem. Phys. 121, 1187 (2004).

${ }^{85}$ O. A. Vydrov, J. Heyd, A. V. Krukau, and G. E. Scuseria, J. Chem. Phys. 125, 074106 (2006).

${ }^{86}$ O. A. Vydrov and G. E. Scuseria, J. Chem. Phys. 125, 234109 (2006).

${ }^{87}$ O. A. Vydrov, G. E. Scuseria, and J. P. Perdew, J. Chem. Phys. 126, 154109 (2007).

${ }^{88}$ Y. Zhao and D. G. Truhlar, Theor. Chem. Acc. 120, 215 (2008).

${ }^{89}$ Y. Zhao and D. G. Truhlar, J. Chem. Phys. 125, 194101 (2006).

${ }^{90}$ R. Peverati and D. G. Truhlar, J. Phys. Chem. Lett. 2, 2810 (2011).

${ }^{91}$ H. S. Yu, X. He, S. L. Li, and D. G. Truhlar, Chem. Sci. 7, 5032 (2016).

${ }^{92}$ H. S. Yu, X. He, and D. G. Truhlar, J. Chem. Theory Comput. 12, 1280 (2016).

${ }^{93}$ C. Adamo and V. Barone, J. Chem. Phys. 108, 664 (1998).

${ }^{94}$ J. P. Perdew, J. A. Chevary, S. H. Vosko, K. A. Jackson, M. R. Pederson, D. J. Singh, and C. Fiolhais, Phys. Rev. B 46, 6671 (1992).

${ }^{95}$ J. P. Perdew, K. Burke, and Y. Wang, Phys. Rev. B 54, 16533 (1996).

${ }^{96}$ X. Xu and W. A. Goddard, Proc. Natl. Acad. Sci. U. S. A. 101, 2673 (2004).

${ }^{97}$ A. J. Cohen and N. C. Handy, Mol. Phys. 99, 607 (2001).

${ }^{98}$ N. C. Handy and A. J. Cohen, Mol. Phys. 99, 403 (2001).

${ }^{99}$ S. H. Vosko, L. Wilk, and M. Nusair, Can. J. Phys. 58, 1200 (1980).

${ }^{100} \mathrm{C}$. Adamo and V. Barone, J. Chem. Phys. 110, 6158 (1999).

${ }^{101}$ Y. Zhao and D. G. Truhlar, J. Phys. Chem. A 109, 5656 (2005).

${ }^{102}$ M. Ernzerhof and J. P. Perdew, J. Chem. Phys. 109, 3313 (1998).

${ }^{103}$ J. P. Perdew, A. Ruzsinszky, G. I. Csonka, L. A. Constantin, and J. Sun, Phys. Rev. Lett. 103, 026403 (2009).

${ }^{104}$ B. Hammer, L. B. Hansen, and J. K. Nørskov, Phys. Rev. B 59, 7413 (1999).

${ }^{105} \mathrm{~J}$. C. Slater, Quantum Theory of Molecular and Solids: The Self-Consistent Field for Molecular and Solids (McGraw-Hill, New York, 1974), Vol. 4.

${ }^{106}$ A. D. Boese and N. C. Handy, J. Chem. Phys. 116, 9559 (2002).

${ }^{107}$ V. N. Staroverov, G. E. Scuseria, J. Tao, and J. P. Perdew, J. Chem. Phys. 119, 12129 (2003).

${ }^{108}$ J.-D. Chai and M. Head-Gordon, Phys. Chem. Chem. Phys. 10, 6615 (2008).

${ }^{109}$ J. Heyd, G. E. Scuseria, and M. Ernzerhof, J. Chem. Phys. 118, 8207 (2003).

${ }^{110}$ R. G. Parr and Z. Zhou, Acc. Chem. Res. 26, 256 (1993).

${ }^{111}$ K. P. Kepp, J. Phys. Chem. A 121, 9092 (2017).

${ }^{112}$ K. A. Moltved and K. P. Kepp, J. Phys. Chem. C 123, 18432 (2019).

${ }^{113}$ T. F. Hughes and R. A. Friesner, J. Chem. Theory Comput. 7, 19 (2011).

${ }^{114}$ M. Reiher, Inorg. Chem. 41, 6928 (2002).

${ }^{115} \mathrm{H}$. Paulsen, L. Duelund, H. Winkler, H. Toftlund, and A. X. Trautwein, Inorg. Chem. 40, 2201 (2001).

${ }^{116}$ K. P. Kepp, in Transition Metals in Coordination Environments: Computational Chemistry and Catalysis Viewpoints, edited by E. Broclawik, T. Borowski, and M. Radoń (Springer International Publishing, 2019), pp. 1-33.

${ }^{117}$ M. Swart, J. Chem. Theory Comput. 4, 2057 (2008).

${ }^{118}$ K. P. Kepp, Commun. Chem. 1, 63 (2018).

${ }^{119}$ O. S. Siig and K. P. Kepp, J. Phys. Chem. A 122, 4208 (2018).

${ }^{120}$ K. A. Moltved and K. P. Kepp, ChemPhysChem 20, 3210 (2019).

${ }^{121}$ M. Mostafanejad, J. Haney, and A. E. DePrince, Phys. Chem. Chem. Phys. 21, 26492 (2019). 